\title{
Influencia de corrosión del acero bajo los elementos de hormigón armado con adiciones anticongelantes
}

\author{
Influence of corrosion of the steel under the elements of reinforced \\ concrete with antifreeze additions
}

\author{
María Eugenia Rollano Quintana \\ maugerollanoquintana@gmail.com \\ Universidad Autónoma Tomas Frías, Bolivia
}

Artículo recibido enero 2019 | Arbitrado en feb-marzo 2019 | Publicado en mayo 2019

\section{RESUMEN}

Las patologías más frecuentes consecuencia de procesos de hormigonado en temperaturas frías son: la fisuración, pérdida de recubrimiento y corrosión de armaduras de acero por incorporación de sales en forma de aditivos anticongelantes, en el proceso de mezclado o posteriores al fraguado de la mezcla. Por tanto, es necesario conocer la influencia de la corrosión del acero producida por adiciones de anticongelantes en la adherencia de elementos de hormigón armado. Para ello se ha llevado a cabo un programa experimental de ensayos tipo pull - out centrado, con probetas de hormigón con aditivos anticongelantes, unas sin corrosión y otras sometidas a procesos de corrosión acelerada, con diferentes grados de deterioro en las barras; mediante las que se ha obtenido un modelo lineal simple, que representa la variación de la tensión de adherencia como función del diámetro de las barras y modelos de regresión polinomial que representan la variación de la tensión de adherencia como función del nivel de corrosión; mediante los cuales se realiza la evaluación de la influencia de la corrosión de armaduras de acero en la adherencia de elementos de hormigón armado con adiciones de anticongelantes.

Palabras clave: Aditivos anticongelantes; corrosión; adherencia; hormigón armado, ensayo pull-out

\section{ABSTRACT}

The most frequent pathologies consequence of concreting processes in cold temperatures are: cracking, loss of coating and corrosion of steel reinforcements due to the incorporation of salts in the form of antifreeze additives, in the mixing process or after the mixture has set. Therefore, it is necessary to know the influence of steel corrosion produced by antifreeze additions on the adherence of reinforced concrete elements. For this, an experimental program of centered pull-out type tests has been carried out, with concrete specimens with antifreeze additives, some without corrosion and others subjected to accelerated corrosion processes, with different degrees of deterioration in the bars; by means of which a simple linear model has been obtained, which represents the variation of the bond stress as a function of the diameter of the bars and polynomial regression models that represent the variation of the bond stress as a function of the level of corrosion; through which the evaluation of the influence of the corrosion of steel reinforcements on the adherence of reinforced concrete elements with antifreeze additions is carried out.

Key words: Antifreeze additives; corrosion; adhesion; reinforced concrete, pull-out test 


\section{INTRODUCCIÓN}

En países con clima continental como Bolivia, el congelamiento del agua en los poros del hormigón es causa frecuente de deterioro del hormigón; pues las condiciones ambientales y mecánicas a las que están sometidas las estructuras son modificadas en el hormigonado y a lo largo de su vida útil. Las patologías más frecuentes producto de procesos de hormigonado en temperaturas frías de estructuras de hormigón armado son la fisuración, pérdida de recubrimiento y corrosión de las armaduras de acero por incorporación de sales en el proceso de mezclado o posteriores al fraguado de la mezcla.

El hormigón armado, uno de los principales materiales usados en la construcción, emplea armaduras de acero para aportar propiedades de resistencia a la tracción, evitando fallos en las estructuras de hormigón que están sujetas a variedad de esfuerzos; el aporte de las armaduras se produce por la transferencia de tensiones por adherencia; siendo los mecanismos resistentes que se movilizan en la adherencia básicamente tres: la adhesión química, el rozamiento y la interacción mecánica entre el acero y el hormigón.

El estudio de la pérdida de adherencia es un factor a considerar en la capacidad de carga de una estructura. Debido a ella, la transferencia de tensiones no se produce correctamente, y consecuentemente, el comportamiento entre hormigón y acero se deteriora de tal manera que las barras no son capaces de asumir la tensión que les corresponde. Ésta pérdida de la adherencia es el resultado de varios factores entre ellos el nivel de corrosión de las armaduras.

Bolivia es un país caracterizado por la variedad de climas que experimenta, las temperaturas más bajas se producen en el extremo oeste (La Paz, Oruro y Potosí) con promedio anual de $-7^{\circ} \mathrm{C}$ y temperaturas mínimas medias con valores entre $-5^{\circ} \mathrm{C}$ y $3^{\circ} \mathrm{C}$ (SENAMHI, 2013).

En estas condiciones se emplean de forma difundida aditivos anticongelantes que por su composición, favorecen notablemente el desarrollo de la corrosión de metales entre los cuales se incluye las barras de acero en las estructuras de hormigón armado.

Considerando que una de las principales causas de deterioro en las estructuras de hormigón armado es la corrosión de la armadura, fenómeno que afecta al acero por reducción de su sección y cambios en sus propiedades mecánicas, y al hormigón por fisuración producto de la presión radial que ejercen los óxidos; es un fenómeno que influye en la transmisión de tensiones entre hormigón y acero por pérdida de adherencia.

El comportamiento resistente de las estructuras de hormigón armado se fundamenta en una adecuada transferencia de tensiones entre hormigón y acero, si este mecanismo se deteriora se afectaría la integridad de los elementos, modificando de forma negativa la capacidad resistente de las estructuras, su grado de seguridad $\mathrm{y}$ durabilidad. Basado en la problemática de este estudio se va dar respuesta a ¿Cómo influye la corrosión del acero producida por adiciones de anticongelantes en la adherencia de elementos de hormigón armado? Esto con el propósito de evaluar la influencia de la corrosión de armaduras de acero en la adherencia de elementos de hormigón armado con adiciones de anticongelantes. Para así, lograr definir los grados de corrosión en barras de acero de refuerzo, a partir del proceso de corrosión acelerada, mediante el método galvanostático. Al mismo tiempo de obtener diagramas de tensión de adherencia - deslizamiento en elementos de hormigón armado con adiciones de anticongelantes, para grados de 
corrosión con penetraciones promedio menores a $350 \mathrm{~mm}$., en barras de acero de diámetro: 9,5 mm, $12 \mathrm{~mm}, 16 \mathrm{~mm}, 20 \mathrm{~mm}$ y $25 \mathrm{~mm}$, a partir de ensayos de caracterización de adherencia hormigónacero tipo pull-out. Con la finalidad de evaluar el comportamiento de la adherencia entre acero y hormigón a partir de la caracterización de la influencia de la corrosión de barras de acero en hormigones armados con adiciones de anticongelantes.

La importancia de este estudio radica en que la corrosión de las armaduras de acero constituye uno de los mecanismos de deterioro detectados con más frecuencia en estructuras de hormigón armado. Cuando agentes desencadenantes de la corrosión, como ser adiciones de anticongelantes entran en contacto con la armadura, se provoca la despasivación de la misma, iniciando el proceso de deterioro progresivo que afecta las características de los materiales constitutivos del hormigón armado, así como a los mecanismos de interacción entre ambos materiales: adherencia.

\section{La adherencia}

La adherencia es el mecanismo de interacción que permite la transferencia de fuerza entre las barras de refuerzo y el hormigón circundante, asegurando la acción compuesta entre los dos materiales (Hernández y Hernández, 2010); se constituye en una de las cualidades más representativas del hormigón armado, porque mediante ella es posible considerar el trabajo conjunto de hormigón y acero, a pesar de que sus materiales componentes posean propiedades y características diferentes.

La adherencia desempeña dos roles principales: anclar las barras de acero en la masa de hormigón y asegurar una eficiente transmisión de los esfuerzos tangenciales que se producen en el perímetro de la barra. (Hernández y Hernández, 2010).

Estos esfuerzos son producidos cuando una barra embebida en una matriz de hormigón es sometida a una fuerza de tracción y ocasiona la transferencia de tensiones al hormigón, produciendo fuerzas de compresión inclinadas originadas en las corrugas, según un ángulo a (Figura 1). La componente radial de esta fuerza de compresión se equilibra con un anillo de tracciones que aparece en el hormigón rodeando a la barra. (Tepfers, 1973).

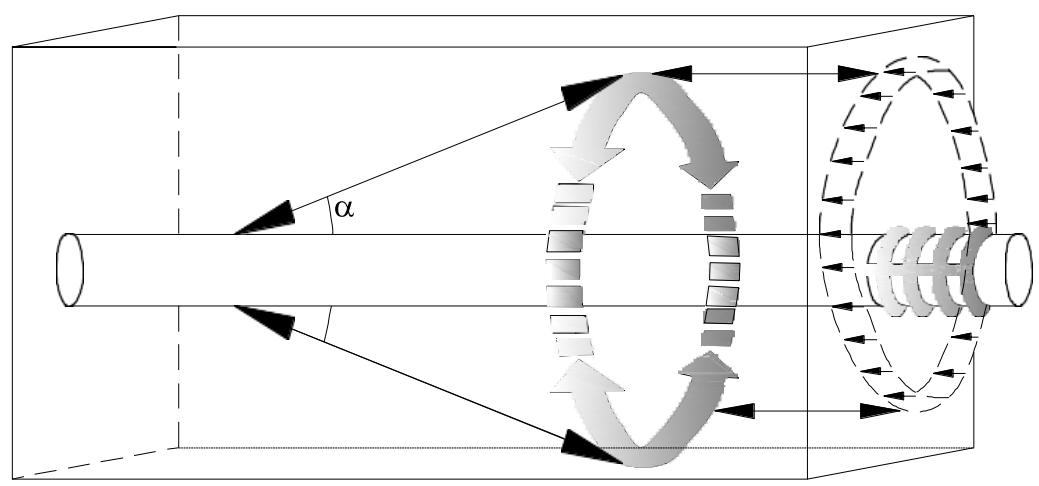

Figura 1. Efecto de los componentes radiales de las fuerzas de adherencia 
A theory of bond applied to overlapped tensile reinforcement splices for deformed bars (Tepfers, 1973).

La transferencia de tensiones entre la armadura de acero y el hormigón que lo rodea se produce a través de los siguientes mecanismos, explicados en la figura 2:

- Adhesión química, debida a la interconexión físico-química de partículas de la pasta de cemento con la rugosidad de la superficie de contacto de la armadura, vinculan al acero con la pasta cementicia por medio del efecto de retracción de fraguado. (Ruiz et al., 2012)

- Rozamiento, producido cuando se pierde el mecanismo de adhesión química y es debido principalmente a las características e irregularidades de la barra.(Delgado, 2008)

- Interacción mecánica, producida por el incremento en el deslizamiento de la barra debido a la fisuración interna, por lo que se debe principalmente al confinamiento, recubrimiento, armadura transversal, nivel de tensión entre otros. (Lundgren, 2007; Simões, 2013).

\section{MATERIALES Y MÉTODOS}

En este apartado es descrito el programa experimental de ensayos tipo pull
- out: el principio de funcionamiento del ensayo, las propiedades de los materiales empleados en la fabricación de las probetas y su geometría, los equipos necesarios para su instrumentación y finalmente, los resultados que se obtuvieron para evaluar la influencia del diámetro de la barra de acero y el nivel de corrosión en la relación adherencia local-deslizamiento y en la tensión máxima de adherencia.

\section{Programa de ensayos}

Se desarrollan cinco series de ensayos, cada serie contempla un diámetro nominal de barra: la serie 1 corresponde a barras de 9,5 $\mathrm{mm}$. de diámetro, la serie 2 a barras de $12 \mathrm{~mm}$. de diámetro, la serie 3 a barras de $16 \mathrm{~mm}$. de diámetro, la serie 4 a barras de $20 \mathrm{~mm}$. de diámetro y por último la serie 5 a barras de $25 \mathrm{~mm}$. de diámetro; siendo estos diámetros los más empleados en la construcción de edificaciones. Cada serie de probetas tiene cinco variaciones en el nivel de corrosión de las barras, con diferentes pérdidas de área debido a la corrosión acelerada de estas. Así con cinco diámetros nominales y cinco niveles de corrosión, se llega a un total de 25 probetas, identificados en la Tabla 1.

Tabla 1. Identificación de las probetas de ensayo

\begin{tabular}{|c|c|c|c|c|c|}
\hline \multirow[b]{2}{*}{ Nivel de corrosión } & \multicolumn{5}{|c|}{ Diámetro de barras } \\
\hline & f $9,5 \mathrm{~mm}$ & f $12 \mathrm{~mm}$ & f $16 \mathrm{~mm}$ & f $20 \mathrm{~mm}$ & f $25 \mathrm{~mm}$ \\
\hline N1 & M - 1 & M - 2 & M - 3 & M - 4 & M - 5 \\
\hline N2 & M - 6 & M - 7 & M - 8 & M - 9 & $M-10$ \\
\hline N3 & M - 11 & M - 12 & $\mathrm{M}-13$ & M - 14 & M - 15 \\
\hline N4 & M - 16 & M - 17 & M - 18 & M - 19 & M - 20 \\
\hline N5 & M - 21 & M - 22 & M - 23 & M - 24 & M - 25 \\
\hline
\end{tabular}




\section{Propiedades de los materiales}

\section{Hormigón}

La calidad del hormigón se seleccionó bajo los siguientes criterios: ser un hormigón convencional (que tenga resistencia semejante a los que se aplican en estructuras del país), se considera un hormigón con resistencia de $21 \mathrm{MPa}$. En la fabricación de las probetas de todas las series se empleó la siguiente dosificación: Cemento tipo IP - $350 \mathrm{~kg} / \mathrm{m}^{3}$, grava rodada (3/4 pulg.) $-1300 \mathrm{~kg} / \mathrm{m}^{3}$, arena (0,2 pulg.) $-600 \mathrm{~kg} / \mathrm{m}^{3}$, agua $-160 \mathrm{l} / \mathrm{m}^{3}$.

La presencia de las adiciones de anticongelantes se simuló añadiendo el 3\% de cloruros (cloruro de sodio $\mathrm{NaCl}$ ) por peso de cemento, durante el hormigonado. (García, 1998; Li y Zheng, 2005; Tórres, 2011; Zandi et al., 2010). La adición de cloruros además asegura la despasivación de las armaduras, iniciando el proceso corrosivo en las barras de acero.
La calidad del hormigón de las probetas fue controlada mediante ensayos de resistencia a compresión, en probetas cilíndricas de 6 pulg. $(15 \mathrm{~cm})$ de diámetro y 12 pulg. $(30 \mathrm{~cm})$ de altura, sometidas a iguales procesos de vibrado y curado que las probetas prismáticas a ser ensayadas por adherencia; obteniendo una resistencia promedio de $208.4 \mathrm{~kg} / \mathrm{cm}^{2}$.

\section{Armaduras de acero}

Las barras de acero empleadas son corrugadas y de distribución nacional, producidas por ArcelorMittal, conforme a la Norma Brasileña NBR 7480/07 grado CA50S, con las siguientes características mecánicas:

- Límites de Fluencia $\mathrm{f}_{\mathrm{yk}}=500 \mathrm{MPa}$

- Resistencia a tracción $\mathrm{f}_{\mathrm{tr}}=660 \mathrm{MPa}$

Los diámetros de barras de acero empleados tienen las características geométricas indicadas en la Tabla 2.

Tabla 2. Características geométricas de barras de acero ArcelorMittal

\begin{tabular}{ccc}
\hline Diámetro (mm) & Peso métrico $\mathbf{( k g} / \mathbf{m})$ & Sección nominal $\left(\mathbf{m m}^{\mathbf{2}}\right)$ \\
\hline 9,5 & 0,556 & 71,0 \\
12,0 & 0,888 & 113,1 \\
16,0 & 1,578 & 201,1 \\
20,0 & 2,466 & 314,2 \\
25,0 & 3,583 & 490,9 \\
\hline
\end{tabular}

Fuente: Ficha técnica Las Lomas ArcelorMittal (ArcelorMittal, 2016)

\section{Proceso de corrosión acelerada}

Las probetas de ensayo se conectaron a un circuito de corrosión electroquímica por periodos de entre 40 y 130 días (dependiendo del grado de daño nominal por corrosión deseado), para generar la producción acelerada de óxido. Una muestra de cada serie no fue sometida al proceso para ser utilizada como muestra de control.

Para el procedimiento de corrosión acelerada se emplea el método descrito en Prieto (2014), que comprende la aplicación de una corriente eléctrica 
constante mediante un galvanostato a través de un circuito en el que la barra de acero actúa como ánodo, se oxida y los electrones liberados en el proceso circulan a través del hormigón hacia una malla de acero inoxidable situada en la superficie de la probeta de hormigón en la zona de longitud de anclaje de la barras y que actúa como cátodo o contraelectrodo.

Para asegurar que el hormigón actúe como electrolito y favorecer la circulación de electrones, es necesario mantener el hormigón (electrolito) con la humedad suficiente. Para garantizar las condiciones de humedad se coloca una esponja húmeda permanentemente entre la superficie del mismo y la malla de acero.

La densidad de corriente uniforme aplicada en el procedimiento es de 80 $\mu \mathrm{A} / \mathrm{cm}^{2}$; se emplea un galvanostato de múltiples canales para aplicar la densidad deseada a cada serie hasta alcanzar el periodo de tiempo estimado (Tabla 5). El proceso de corrosión acelerada se controla diariamente midiendo las intensidades circundantes en el circuito, mediante una caja de conexión eléctrica. Cuando se alcanzó el valor nominal de corrosión en las barras de cada serie, se dejó de aplicar la corriente anódica y cada probeta fue desconectada del galvanostato, en espera de iniciar la etapa de ensayos de extracción. La estimación de tiempo para el proceso de corrosión se realiza mediante la ley de Faraday para obtener pérdidas de masa entre $3 \%$ y $10 \%$, determinando los tiempos para cada serie expresados en la tabla 5 .

Tabla 5. Tiempos estimados para el procedimiento de corrosión acelerada

\begin{tabular}{cccc}
\hline Serie & Diámetro $(\mathbf{m m})$ & Duración (días) & Pérdida de masa esperada (\%) \\
\hline 1 & 9.5 & $40-85$ & $4-9$ \\
2 & 12 & $40-100$ & $3-9$ \\
3 & 16 & $60-115$ & $4-7$ \\
4 & 20 & $65-130$ & $3-7$ \\
5 & 25 & $70-130$ & $3-6$ \\
\hline
\end{tabular}

\section{Instrumentación y procedimiento de ensayo}

Los ensayos de extracción se realizaron en una prensa hidráulica universal (Alfred J. Amsler y CÍA) de capacidad de 100 Tonf. (Figura 1a); la probeta se sitúa sobre el soporte fijo de la misma (Figura 1b). En cada serie la probeta cúbica de hormigón se apoya en una plancha de goma de dimensiones 25 $\mathrm{cm}$ x $25 \mathrm{~cm}$ y $5 \mathrm{~mm}$ de espesor para evitar el rozamiento entre el apoyo y la probeta, y ésta a su vez sobre una placa de acero de las mismas dimensiones y $1 \mathrm{~cm}$ de espesor para garantizar un apoyo uniforme y el reparto de tensiones sobre la superficie (Figura 1a). Estos elementos tienen una perforación de 2 veces el diámetro la barra a ensayar. La velocidad teórica de aplicación de carga es función del diámetro nominal de la barra de modo que el incremento de la tensión de adherencia sea constante según el ensayo normalizado por la RILEM/CEB/FIP (1983). 
Las velocidades de aplicación de la fuerza de tracción aplicadas fueron: $45 \mathrm{~N} / \mathrm{s}$ (4.59 kgf/s) para la serie $1,72 \mathrm{~N} / \mathrm{s}(7.34$ $\mathrm{kgf} / \mathrm{s}$ ) para la serie $2,128 \mathrm{~N} / \mathrm{s}$ para la serie
3 (13.05 kgf/s), $200 \mathrm{~N} / \mathrm{s}$ (20.39 kgf/s) para la serie 4 y $312 \mathrm{~N} / \mathrm{s}$ para la serie 5 (31.82 $\mathrm{kgf} / \mathrm{s}$ ). a)

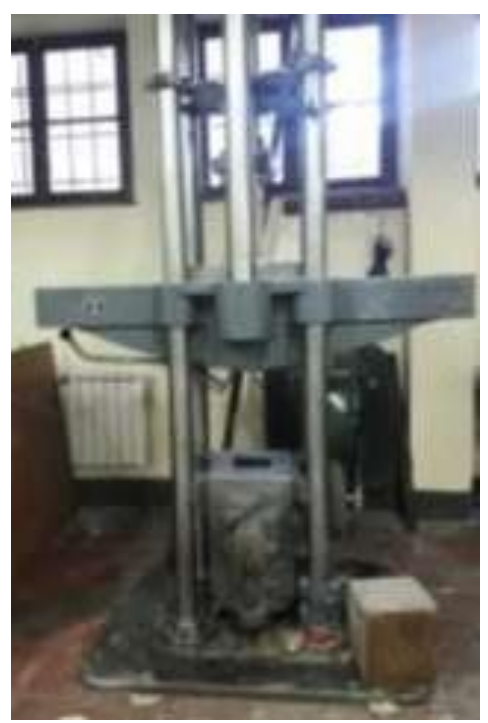

b)

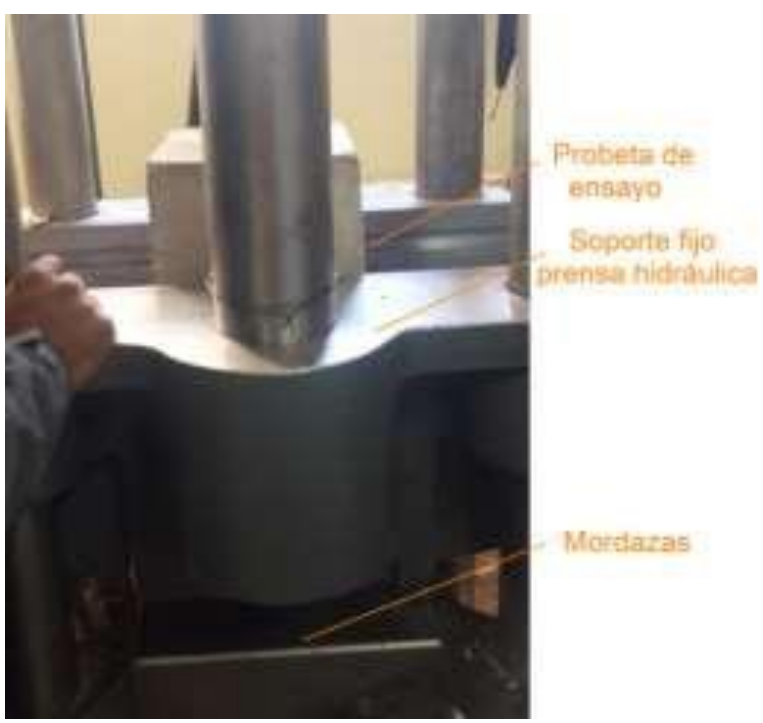

Figura 1. a) Prensa hidráulica universal, b) Posición de la probeta de ensayo

La realización del ensayo comprendió la aplicación de una carga de tracción creciente (a velocidad constante) mediante las mordazas de la prensa hidráulica en el extremo de la barra embebida, registrando el deslizamiento relativo de la barra en el extremo opuesto al de aplicación de la carga (Figura 2 a y b), hasta que ocurre el fallo de adherencia.
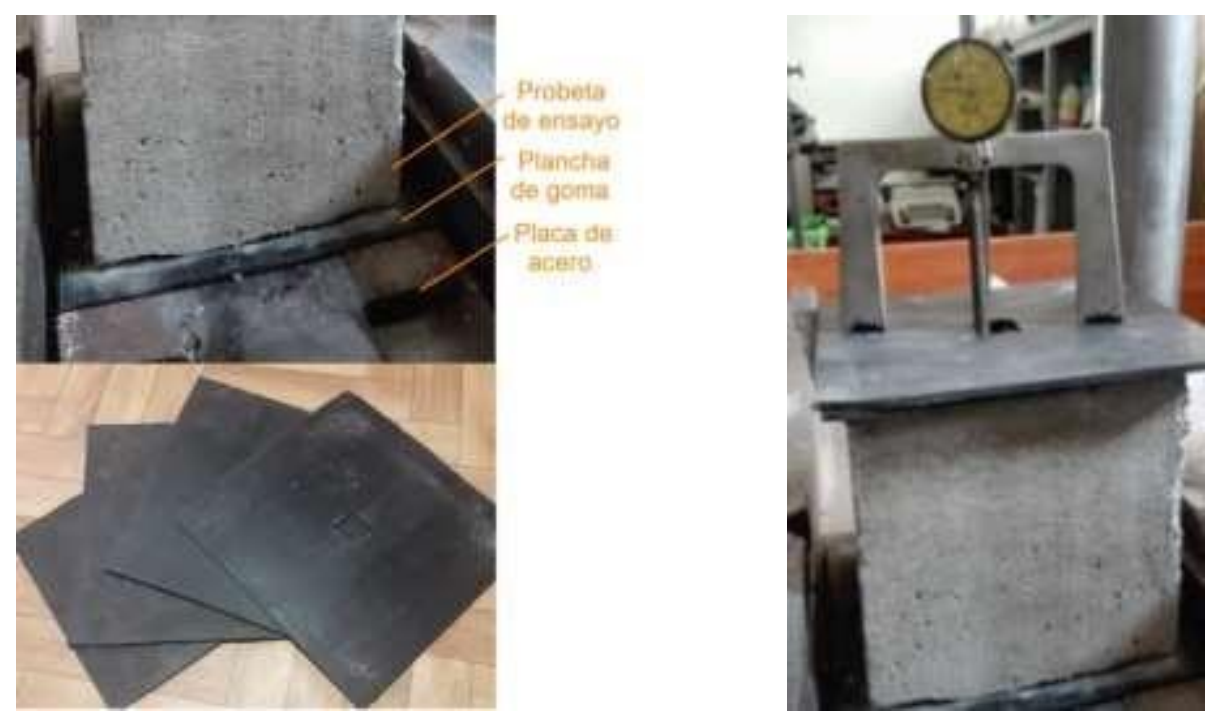

Figura 2. Disposición de ensayo pull - out. a) Placa metálica y plancha de goma de apoyo y b) Desarrollo de uno de los ensayos 
Durante el ensayo se registró la carga aplicada en el extremo activo de la barra, el deslizamiento relativo de la barra respecto al hormigón en el extremo pasivo o cara opuesta a la aplicación de la carga, mediante un dial de deformación con una precisión de 0,01 mm. (Figura 3).

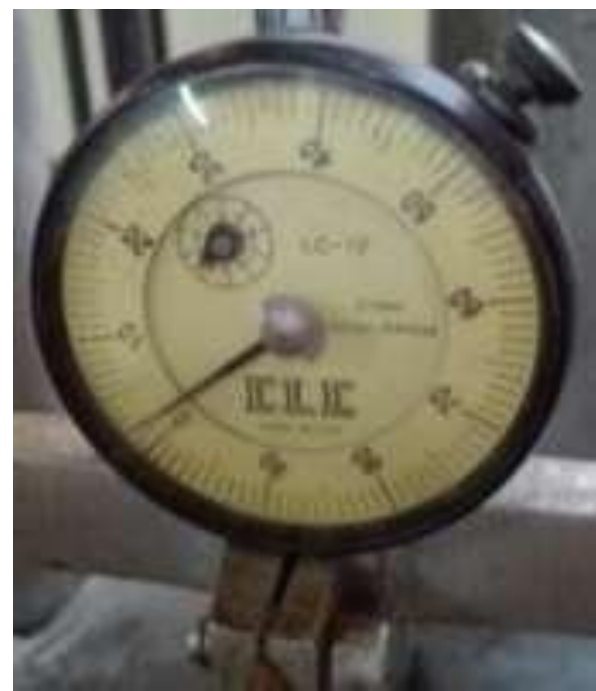

Figura 3. Captador de deslizamiento

\section{Pérdidas corrosión}

Una vez concluidos los procesos de corrosión acelerada y los ensayos de adherencia, la barra de acero fue retirada del hormigón y para conocer el grado de corrosión se elimina el óxido mediante procesos de carácter químico, optando sumergir las barras de acero en una disolución de 100 gramos de ácido oxálico $\left(\mathrm{H}_{2} \mathrm{C}_{2} \mathrm{O}_{4}\right)$ por litro de agua, posteriormente se lavan las barras para eliminar el ácido y se secaron con aire caliente; con este proceso se garantiza que la pérdida del material sólo sea óxido (Figura 4)

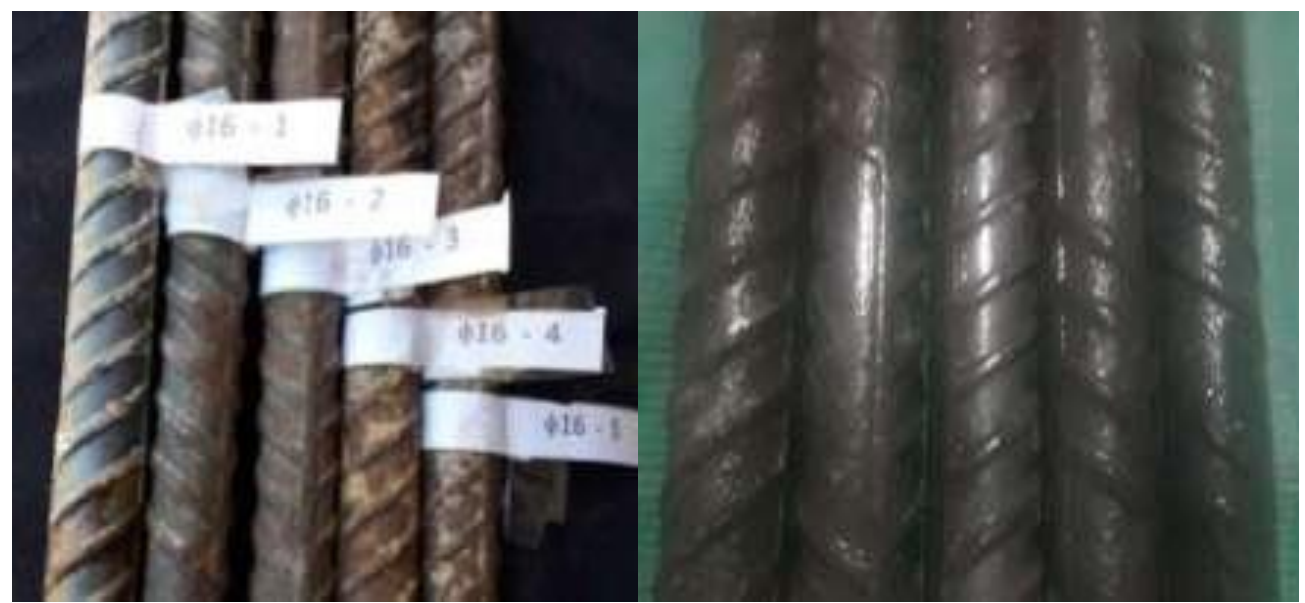

Figura 4. Estado superficial de barras antes y después del proceso de eliminación de óxidos 
Removido el óxido se pesan las barras para después comparar su peso inicial y así obtener el grado de corrosión real.

\section{Resultados del programa experimental}

\section{Pérdidas gravimétricas por corrosión}

Después de la limpieza, la pérdida de masa de las barras $\left(D_{\mathrm{WG}}\right)$ al final del programa experimental fue calculada restándole el valor de la masa final $\left(\mathrm{W}_{\mathrm{f}}\right)$ de la masa inicial $\left(\mathrm{W}_{\mathrm{i}}\right)$. El valor de la penetración promedio por corrosión (ХРRом) (considerando que la corrosión fue uniforme en la zona anódica del refuerzo). (Tabla 6).

Tabla 6. Pérdidas gravimétricas por corrosión

\begin{tabular}{|c|c|c|c|c|c|c|c|}
\hline Serie & Probeta & $\begin{array}{c}\text { Diámetro } \\
\text { de barra } \\
\text { (mm) }\end{array}$ & $\begin{array}{c}\text { Peso } \\
\text { inicial } \\
W_{\text {i }} \text { (gr.) }\end{array}$ & $\begin{array}{c}\text { Peso } \\
\text { final } W_{f} \\
\text { (gr.) }\end{array}$ & $\begin{array}{c}\text { Pérdida } \\
\text { de masa } \\
\mathrm{DW}_{\mathrm{G}}(\mathrm{gr} .)\end{array}$ & $\begin{array}{c}\% \\
\text { pérdida } \\
\text { de masa }\end{array}$ & $\begin{array}{c}\text { Penetración } \\
\text { promedio de } \\
\text { la corrosión } \\
\text { (mm) }\end{array}$ \\
\hline \multirow{5}{*}{1} & M-1 & 9.5 & 389.00 & 388.50 & 0.50 & 0.13 & 3.04 \\
\hline & M-2 & 9.5 & 388.50 & 364.70 & 23.80 & 6.13 & 144.94 \\
\hline & M-3 & 9.5 & 388.00 & 358.70 & 29.30 & 7.55 & 178.43 \\
\hline & M-4 & 9.5 & 387.80 & 356.10 & 31.70 & 8.17 & 193.05 \\
\hline & M-5 & 9.5 & 389.10 & 352.70 & 36.40 & 9.35 & 221.67 \\
\hline \multirow{5}{*}{2} & M-6 & 12 & 621.60 & 620.30 & 1.30 & 0.21 & 6.27 \\
\hline & M-7 & 12 & 620.70 & 598.90 & 21.80 & 3.51 & 105.10 \\
\hline & M-8 & 12 & 620.70 & 589.00 & 31.70 & 5.11 & 152.83 \\
\hline & M-9 & 12 & 619.40 & 578.30 & 41.10 & 6.64 & 198.15 \\
\hline & M-10 & 12 & 621.50 & 566.50 & 55.00 & 8.85 & 265.16 \\
\hline \multirow{5}{*}{3} & M-11 & 16 & 1104.60 & 1103.20 & 1.40 & 0.13 & 5.06 \\
\hline & M-12 & 16 & 1103.80 & 1060.20 & 43.60 & 3.95 & 157.65 \\
\hline & M-13 & 16 & 1104.00 & 1045.50 & 58.50 & 5.30 & 211.53 \\
\hline & M-14 & 16 & 1104.20 & 1038.80 & 65.40 & 5.92 & 236.48 \\
\hline & M-15 & 16 & 1103.90 & 1021.80 & 82.10 & 7.44 & 296.86 \\
\hline \multirow{5}{*}{4} & M-16 & 20 & 1725.80 & 1723.10 & 2.70 & 0.16 & 7.81 \\
\hline & M-17 & 20 & 1725.60 & 1666.90 & 58.70 & 3.40 & 169.80 \\
\hline & M-18 & 20 & 1726.20 & 1653.90 & 72.30 & 4.19 & 209.14 \\
\hline & M-19 & 20 & 1726.00 & 1642.00 & 84.00 & 4.87 & 242.98 \\
\hline & M-20 & 20 & 1726.20 & 1614.70 & 111.50 & 6.46 & 322.53 \\
\hline \multirow{5}{*}{5} & M-21 & 25 & 2507.00 & 2502.90 & 4.10 & 0.16 & 9.49 \\
\hline & M-22 & 25 & 2508.00 & 2433.10 & 74.90 & 2.99 & 173.33 \\
\hline & M-23 & 25 & 2506.50 & 2422.80 & 83.70 & 3.34 & 193.69 \\
\hline & $\mathrm{M}-24$ & 25 & 2507.40 & 2406.70 & 100.70 & 4.02 & 233.03 \\
\hline & M-25 & 25 & 2500.30 & 2358.50 & 141.80 & 5.67 & 328.14 \\
\hline
\end{tabular}

\section{Tensión media de adherencia}

La tensión media de adherencia se calculó como la carga aplicada en el ensayo dividida por la superficie adherente de la barra, suponiendo una distribución de tensiones uniforme. 


\section{Diagramas tensión de adherencia - deslizamiento}

Una vez finalizado los ensayos, se procedió a elaborar los gráficos de tensión de adherencia - deslizamiento, los presentaron en las curvas tensión de adherencia - deslizamiento para las probetas de las cinco series; el último punto de la curva es el punto de falla de la adherencia a partir del cual se produce la extracción de la barra de acero de la probeta de hormigón.

\section{Tensión máxima de adherencia}

Referido a diagramas de tensión de adherencia local - deslizamiento y los valores de tensión máxima de cada serie.

La tabla 7 expresa la carga máxima aplicada en cada ensayo, que corresponde a la tensión máxima de adherencia, el deslizamiento relativo de la barra respecto a la superficie de hormigón para esta tensión y el tipo de falla producido.

Tabla 7. Tensiones máximas de adherencia

\begin{tabular}{|c|c|c|c|c|c|c|}
\hline Serie & Probeta & $\begin{array}{l}\text { barra } \\
(\mathbf{m m})\end{array}$ & $\begin{array}{c}\text { Carga } \\
\text { máxima (kgf) }\end{array}$ & $\begin{array}{l}\text { Tensión Máxima } \\
\text { Adherencia } \\
\text { (MPa) }\end{array}$ & $\begin{array}{l}\text { Deslizamiento } \\
\text { para } t_{\max }(\mathrm{mm})\end{array}$ & Tipo de falla \\
\hline \multirow{5}{*}{1} & $\mathrm{M}-1$ & 9.5 & 3100 & 10.72 & 0.6 & Pull - out \\
\hline & $\mathrm{M}-2$ & 9.5 & 3800 & 13.14 & 0.4 & Pull - out \\
\hline & M-3 & 9.5 & 3550 & 12.28 & 0.7 & Pull - out \\
\hline & M-4 & 9.5 & 3200 & 11.07 & 0.5 & Pull - out \\
\hline & M-5 & 9.5 & 2500 & 8.65 & 0.06 & Pull - out \\
\hline \multirow{5}{*}{2} & M-6 & 12 & 3000 & 6.50 & 0.25 & Pull - out \\
\hline & M-7 & 12 & 3100 & 6.72 & 0.31 & Pull - out \\
\hline & M-8 & 12 & 3800 & 8.24 & 0.65 & Pull - out \\
\hline & M-9 & 12 & 3100 & 6.72 & 0.09 & Pull - out \\
\hline & M-10 & 12 & 2100 & 4.55 & 0.4 & Pull - out \\
\hline \multirow{3}{*}{3} & M-11 & 16 & 3200 & 3.90 & 0.04 & $\begin{array}{l}\text { splitting induced pull } \\
\text { - out }\end{array}$ \\
\hline & M-12 & 16 & 3500 & 4.27 & 0.15 & $\begin{array}{l}\text { splitting induced pull } \\
\text { - out }\end{array}$ \\
\hline & M-13 & 16 & 3100 & 3.78 & 0.05 & Pull - out \\
\hline \multirow{7}{*}{4} & M-14 & 16 & 2900 & 3.54 & 0.04 & Pull - out \\
\hline & M-15 & 16 & 2800 & 3.41 & 0.03 & Pull - out \\
\hline & M-16 & 20 & 2750 & 4.29 & 0.015 & Pull - out \\
\hline & M-17 & 20 & 3000 & 4.68 & 0.12 & Pull - out \\
\hline & M-18 & 20 & 2650 & 4.14 & 0.05 & Pull - out \\
\hline & M-19 & 20 & 2300 & 3.59 & 0.045 & Pull - out \\
\hline & $\mathrm{M}-20$ & 20 & 1600 & 2.50 & 0.05 & $\begin{array}{l}\text { splitting induced pull } \\
\text { - out }\end{array}$ \\
\hline
\end{tabular}




\begin{tabular}{ccccccc}
\hline Serie & Probeta & $\begin{array}{c}\text { Q barra } \\
(\mathbf{m m})\end{array}$ & $\begin{array}{c}\text { Carga } \\
\text { máxima (kgf) }\end{array}$ & $\begin{array}{c}\text { Tensión Máxima } \\
\text { Adherencia } \\
\text { (MPa) }\end{array}$ & $\begin{array}{c}\text { Deslizamiento } \\
\text { para } \mathbf{t}_{\text {max }}(\mathbf{m m})\end{array}$ & Tipo de falla \\
\hline & & & & & \\
\hline & M-21 & 25 & 3500 & 3.50 & 0.02 & Pull - out \\
& M-22 & 25 & 3750 & 3.75 & 0.08 & Pull - out \\
& M-23 & 25 & 3700 & 3.70 & 0.02 & Pull - out \\
& M-24 & 25 & 3100 & 3.10 & 0.05 & Pull - out \\
& M-25 & 25 & 2800 & 2.80 & 0.02 & Pull - out \\
\hline
\end{tabular}

\section{RESULTADOS}

Evaluación del comportamiento adherente hormigón - acero en elementos de hormigón armado con adiciones de anticongelantes

Se muestran los resultados de los ensayos de adherencia con armaduras sanas y corroídas. Para el análisis se emplearon modelos de regresión simple y regresión polinomial, modelos estadísticos que describieron el impacto de un factor cuantitativo (deslizamiento d y penetración promedio de corrosión) sobre una variable dependiente (tensión de adherencia $\mathrm{t} \mathrm{y}$ tensión máxima de adherencia $t_{\max }$ ), que se resuelven por el método de mínimos cuadrados ordinarios. La aplicación de estos modelos se realiza empleando el programa estadístico STHATGRAPHICS 17.1. Además se evaluó el proceso de corrosión de la armadura por adiciones de anticongelantes y su influencia en el valor de la longitud de desarrollo.

Influencia de la degradación de la adherencia en la capacidad resistente de elementos de hormigón armado

El diseño estructural del hormigón armado se fundamentó en hipótesis y condiciones de equilibrio y compatibilidad; una que merece especial atención es: entre el hormigón y el acero debe existir una perfecta adherencia tal que se garanticen iguales deformaciones en los dos materiales bajo la acción de las cargas. Es por tanto, la adherencia un fenómeno en el que se fundamenta el comportamiento del hormigón armado. La adherencia, idealmente considerada como perfecta; se ve afectada por situaciones como la incorporación de anticongelantes en el amasado, que crean situaciones críticas que originan la degradación de este fenómeno y por consiguiente puede afectar la durabilidad y modificar la respuesta del sistema estructural en su conjunto.

La capacidad resistente de estructuras de hormigón armado se fundamentó en una adecuada transferencia de tensiones entre hormigón y acero; cuando existe una degradación de la adherencia, el mecanismo de transferencia de tensiones se deteriora, modificando negativamente la capacidad resistente; incrementa su deformación y disminuye su seguridad. (M. Fernández, 2003; Molina y Gutiérrez, 2008).

En los elementos a flexión, la posición y el grado de deterioro de la adherencia, se produce una reducción de la carga de rotura y un aumento de la deformación bajo cargas de servicio. Si el deterioro de la adherencia se localiza en zonas de anclaje o solapo, puede desencadenar el colapso. Investigaciones realizadas ponen de 
manifiesto que la capacidad residual de vigas disminuye considerablemente $(10 \%$ al $20 \%$ ) a partir de $30 \%$ de pérdida de adherencia de la armadura traccionada. (Molina y Gutiérrez, 2008).

\section{Fallos por adherencia y anclaje}

Los síntomas patológicos que afectaran a las estructuras de hormigón armado, producto de una reducción de la adherencia hormigón - acero, son frecuentemente expresados como fisuras. En la Figura 5 se presenta un esquema de fisuración típica por deficiencias en la transmisión de tensiones hormigón - acero.

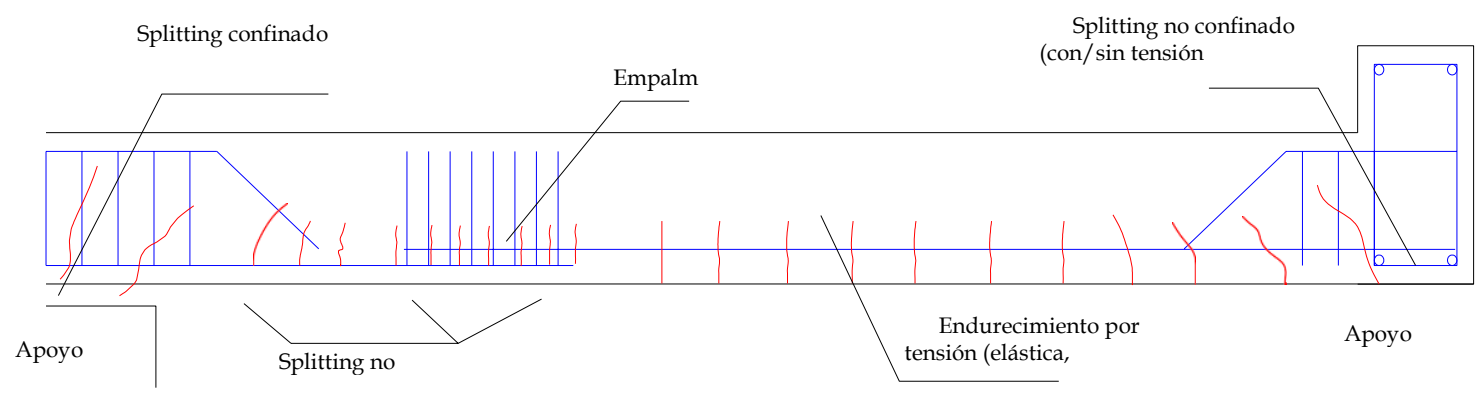

Figura 5. Fisuración típica por deficiente transmisión de tensiones hormigón - acero Bond of reinforcement in concrete (Fib, 2000).

Los fallos por adherencia y anclaje se manifiestan mediante fisuras localizadas paralelas a las barras principales; la armadura deja de colaborar con el hormigón, ésta se desliza y pierde su eficacia.

La rotura por anclaje tiene un carácter frágil, pudiendo sobrevenir en cualquier instante, siendo los posibles fallos de anclaje: rotura de la adherencia (se produce en barras lisas, hormigón de baja resistencia o longitudes de anclaje insuficientes), rotura del hormigón (se produce en barras corrugadas, hormigones de baja resistencia) o rotura a tracción de la armadura.

\section{Análisis de resultados}

En las curvas tensión de adherencia local - deslizamiento hasta la pérdida total de adherencia, se determinó las diferentes fases del mecanismo de adherencia: una primera rama casi vertical donde no se producen deslizamientos, una rama tipo parabólica hasta la tensión máxima de adherencia y un pequeño tramo casi horizontal. (Figura 6). 


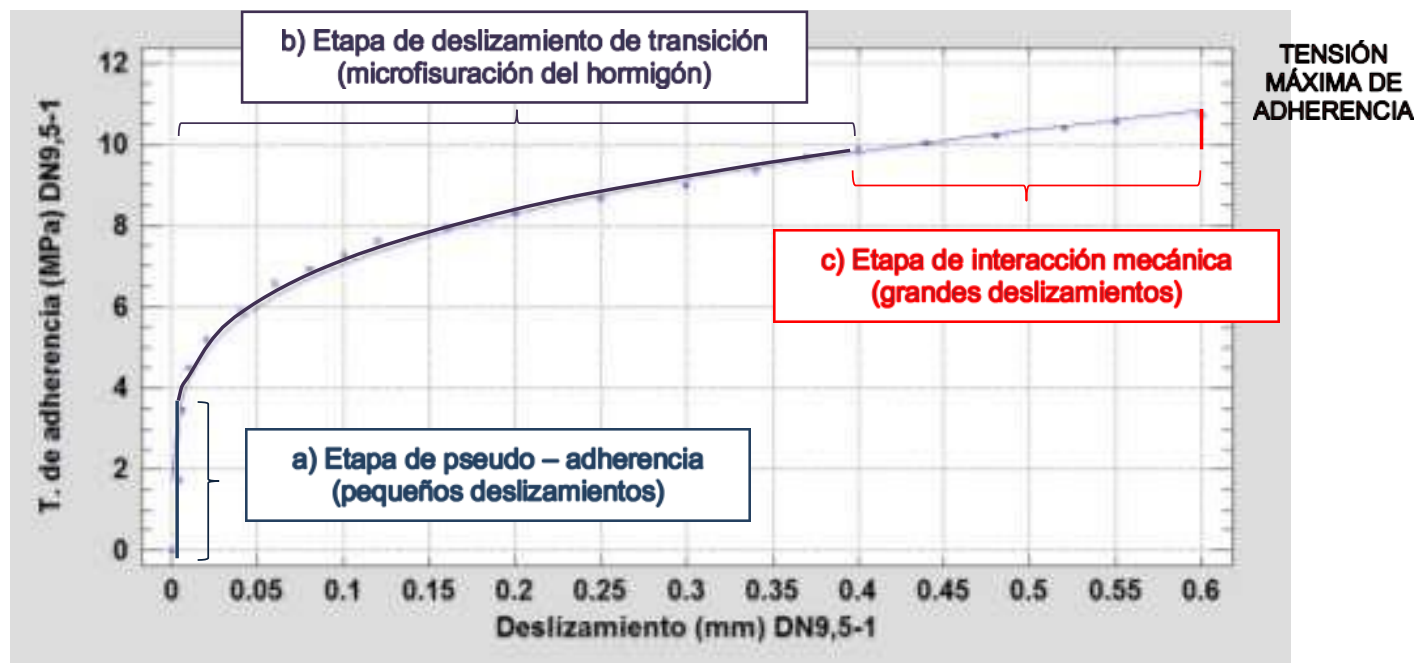

Figura 6. Fases del comportamiento adherente (Ensayo M-1) Elaboración propia

El tramo inicial o etapa de pseudo adherencia, asociada a la adherencia química; seguidamente se mostró en el tramo parabólico o etapa de deslizamiento de transición, provocados por el microagrietamiento inducido por el bloqueo mecánico creado por la presencia de corrugas de la barra de acero, se caracteriza por el aplastamiento local y la microfisuración del hormigón; finalmente se produce un tramo con grandes deslizamientos asociados al mecanismo de interacción mecánica (producida por el incremento en el deslizamiento de la barra debido a la fisuración interna), este tramo se desarrolla hasta la tensión máxima de adherencia.

\section{Modelo propuesto de tensión de adherencia - deslizamiento}

En la tabla 8 se muestran los resultados de procedimientos de regresión simple para la construcción de modelos estadísticos que describan el impacto del deslizamiento (mm.) sobre la tensión de adherencia (MPa.). Los modelos son ajustados usando el procedimiento de mínimos cuadrados. Además, se muestra el tipo modelo que describe e l comportamiento, la ecuación del modelo y los parámetros estadísticos que determinan la significancia estadística del modelo. E $\mathrm{n}$ términos generales, este tipo de modelo capta el comportamiento de la adherencia: una rigidez inicial correspondiente a un material no dañado (sin presencia de fisuras); una rigidez intermedia caracterizada por la propagación de macrofracturas por tracción; por último, una recta horizontal que define el mecanismo de falla límite, por haberse alcanzado la tensión de adherencia máxima. 
Tabla 8. Modelos propuestos tensión de adherencia - deslizamiento

\begin{tabular}{|c|c|c|c|c|c|c|c|}
\hline Experimento & $\begin{array}{l}\text { Modelo que describe el } \\
\text { comportamiento }\end{array}$ & Modelo & Valor $\mathbf{P}$ & $\begin{array}{c}\text { R- cuadrada } \\
(\%)\end{array}$ & $\begin{array}{l}\text { Coeficiente de } \\
\text { correlación }\end{array}$ & $\begin{array}{l}\text { Error } \\
\text { estándar }\end{array}$ & $\begin{array}{c}\text { Error } \\
\text { Absoluto } \\
\text { medio } \\
\end{array}$ \\
\hline$M-1$ & Cuadrado-Y Raíz Cuadrada-X & $\sqrt{\sqrt{ }}$ & -0.0000 & 99.2852 & 0.99642 & 3.17306 & 2.35348 \\
\hline$M-2$ & Cuadrado-Y Raíz Cuadrada-X & $\sqrt{\sqrt{ }}$ & -0.0000 & 95.4276 & 0.97687 & 12.5468 & 10.7524 \\
\hline$M-3$ & Cuadrado-Y Raíz Cuadrada-X & $\sqrt{\sqrt{ }}$ & -0.0000 & 99.4037 & 0.997014 & 3.75121 & 2.58582 \\
\hline$M-4$ & Cuadrado-Y Raíz Cuadrada-X & $\overline{\sqrt{ }}$ & -0.0000 & 99.2130 & 0.996057 & 3.56755 & 2.71149 \\
\hline M-5 & Cuadrado-Y Raíz Cuadrada-X & $\sqrt{\sqrt{ }}$ & -0.0000 & 96.3801 & 0.981734 & 4.78205 & 3.74596 \\
\hline M-6 & Cuadrado-Y Raíz Cuadrada-X & $\sqrt{\sqrt{ }}$ & -0.0000 & 98.2530 & 0.991226 & 1.91894 & 1.45159 \\
\hline$M-7$ & Cuadrado-Y Raíz Cuadrada-X & $\sqrt{\sqrt{ }}$ & -0.0000 & 96.6627 & 0.983172 & 2.86296 & 2.31133 \\
\hline$M-8$ & Cuadrado-Y Raíz Cuadrada-X & $\sqrt{\sqrt{ }}$ & -0.0000 & 82.1974 & 0.906628 & 9.53034 & 7.76018 \\
\hline M-9 & Cuadrado-Y Raíz Cuadrada-X & $\sqrt{\sqrt{ }}$ & 0.0000 & 89.3832 & 0.945427 & 4.65557 & 3.71838 \\
\hline
\end{tabular}




\begin{tabular}{|c|c|c|c|c|c|c|c|}
\hline Experimento & $\begin{array}{l}\text { Modelo que describe el } \\
\text { comportamiento }\end{array}$ & Modelo & Valor $\mathbf{P}$ & $\begin{array}{c}\text { R- cuadrada } \\
\text { (\%) }\end{array}$ & $\begin{array}{l}\text { Coeficiente de } \\
\text { correlación }\end{array}$ & $\begin{array}{l}\text { Error } \\
\text { estándar }\end{array}$ & $\begin{array}{c}\text { Error } \\
\text { Absoluto } \\
\text { medio } \\
\end{array}$ \\
\hline$M-11$ & Cuadrado-Y Raíz Cuadrada-X & $\sqrt{\sqrt{ }}$ & -0.0000 & 91.7891 & 0.958066 & 1.46535 & 1.10565 \\
\hline $\mathrm{M}-12$ & Cuadrado-Y Raíz Cuadrada-X & $\overline{\sqrt{ }}$ & -0.0000 & 72.6405 & 0.852294 & 3.22613 & 2.78937 \\
\hline$M-13$ & Cuadrado-Y Raíz Cuadrada-X & $\sqrt{\sqrt{ }}$ & -0.0000 & 81.0910 & 0.900505 & 2.23129 & 1.89901 \\
\hline$M-14$ & Cuadrado-Y Raíz Cuadrada-X & $\sqrt{\sqrt{ }}$ & 0.0000 & 81.6571 & 0.903643 & 1.82087 & 1.37033 \\
\hline$M-15$ & Cuadrado-Y Raíz Cuadrada-X & $\overline{\sqrt{ }}$ & 0.0000 & 85.2967 & 0.923562 & 1.46673 & 1.08002 \\
\hline$M-16$ & Cuadrado-Y Raíz Cuadrada-X & $\overline{\sqrt{ }}$ & 0.0000 & 86.4509 & 0.92979 & 2.47731 & 1.84318 \\
\hline$M-17$ & Cuadrado-Y Raíz Cuadrada-X & $\sqrt{\sqrt{ }}$ & 0.0000 & 82.2746 & 0.907053 & 2.95645 & 2.49057 \\
\hline $\mathrm{M}-18$ & Cuadrado-Y Raíz Cuadrada-X & $\overline{\sqrt{ } \sqrt{ }}$ & 0.0000 & 91.2485 & 0.955241 & 1.80029 & 1.51825 \\
\hline
\end{tabular}




\begin{tabular}{|c|c|c|c|c|c|c|c|}
\hline Experimento & $\begin{array}{l}\text { Modelo que describe el } \\
\text { comportamiento }\end{array}$ & Modelo & Valor $\mathbf{P}$ & $\begin{array}{c}\text { R- cuadrada } \\
\text { (\%) }\end{array}$ & $\begin{array}{l}\text { Coeficiente de } \\
\text { correlación }\end{array}$ & $\begin{array}{l}\text { Error } \\
\text { estándar }\end{array}$ & $\begin{array}{c}\text { Error } \\
\text { Absoluto } \\
\text { medio } \\
\end{array}$ \\
\hline M-19 & Cuadrado-Y Raíz Cuadrada-X & $\sqrt{\sqrt{ }}$ & 0.0000 & 89.8370 & 0.947824 & 1.50528 & 1.16076 \\
\hline$M-20$ & Cuadrado-Y Raíz Cuadrada-X & $\sqrt{\sqrt{ }}$ & -0.0000 & 88.5762 & 0.941149 & 0.735076 & 0.598764 \\
\hline$M-21$ & Cuadrado-Y Raíz Cuadrada-X & $\sqrt{\sqrt{ }}$ & -0.0000 & 82.6239 & 0.908977 & 1.83518 & 1.54463 \\
\hline$M-22$ & Cuadrado-Y Raíz Cuadrada-X & $\sqrt{\sqrt{ }}$ & -0.0000 & 69.7924 & 0.835419 & 2.76954 & 2.40068 \\
\hline$M-23$ & Cuadrado-Y Raíz Cuadrada-X & $\sqrt{\sqrt{ }}$ & 0.0000 & 83.2412 & 0.912366 & 1.98893 & 1.71604 \\
\hline$M-24$ & Cuadrado-Y Raíz Cuadrada-X & $\overline{\sqrt{ }}$ & -0.0000 & 89.1212 & 0.944041 & 1.09052 & 0.820084 \\
\hline$M-25$ & Cuadrado-Y Raíz Cuadrada-X & $\sqrt{\sqrt{ }}$ & -0.0000 & 81.7797 & 0.904321 & 1.15268 & 0.86266 \\
\hline
\end{tabular}

$\mathrm{t}=$ Tensión de adherencia, MPa.

$\mathrm{d}=$ Deslizamiento, $\mathrm{mm}$.

Elaboración propia basada en resultados de STHATGRAPHICS 17.1 


\section{Tensión de adherencia - diámetro}

Se analizaron la influencia del diámetro de la armadura $(9.5,12,16,20$ y $25 \mathrm{~mm}$.) en la tensión de adherencia; previamente se verifica si existe una relación significativa entre ambos.

\section{Tensión de adherencia (sin corrosión acelerada) - diámetro}

Mediante procedimientos de regresión simple se construyó un modelo estadístico que describe el impacto del diámetro de la barra (mm) sobre la tensión de adherencia (MPa.) de barras de acero que no fueron sometidas a procesos de corrosión acelerada. Una vez omitida la tensión de adherencia correspondiente a M-11 que corresponde a la barra de diámetro $16 \mathrm{~mm}$, se ajusta el modelo representado en la Figura 8.

Los parámetros estadísticos indican una mejor correlación entre la tensión de adherencia y el diámetro de la barra con un nivel de confianza de $95.0 \%$ (Valor $\mathrm{P}=0.0071$, coeficiente de correlación= 0.993). Mediante el modelo ajustado se calcula el valor de tensión de adherencia omitido del modelo; siendo la tensión de adherencia (sin corrosión acelerada) igual a $5.23 \mathrm{MPa}$. para un diámetro de $16 \mathrm{~mm}$.

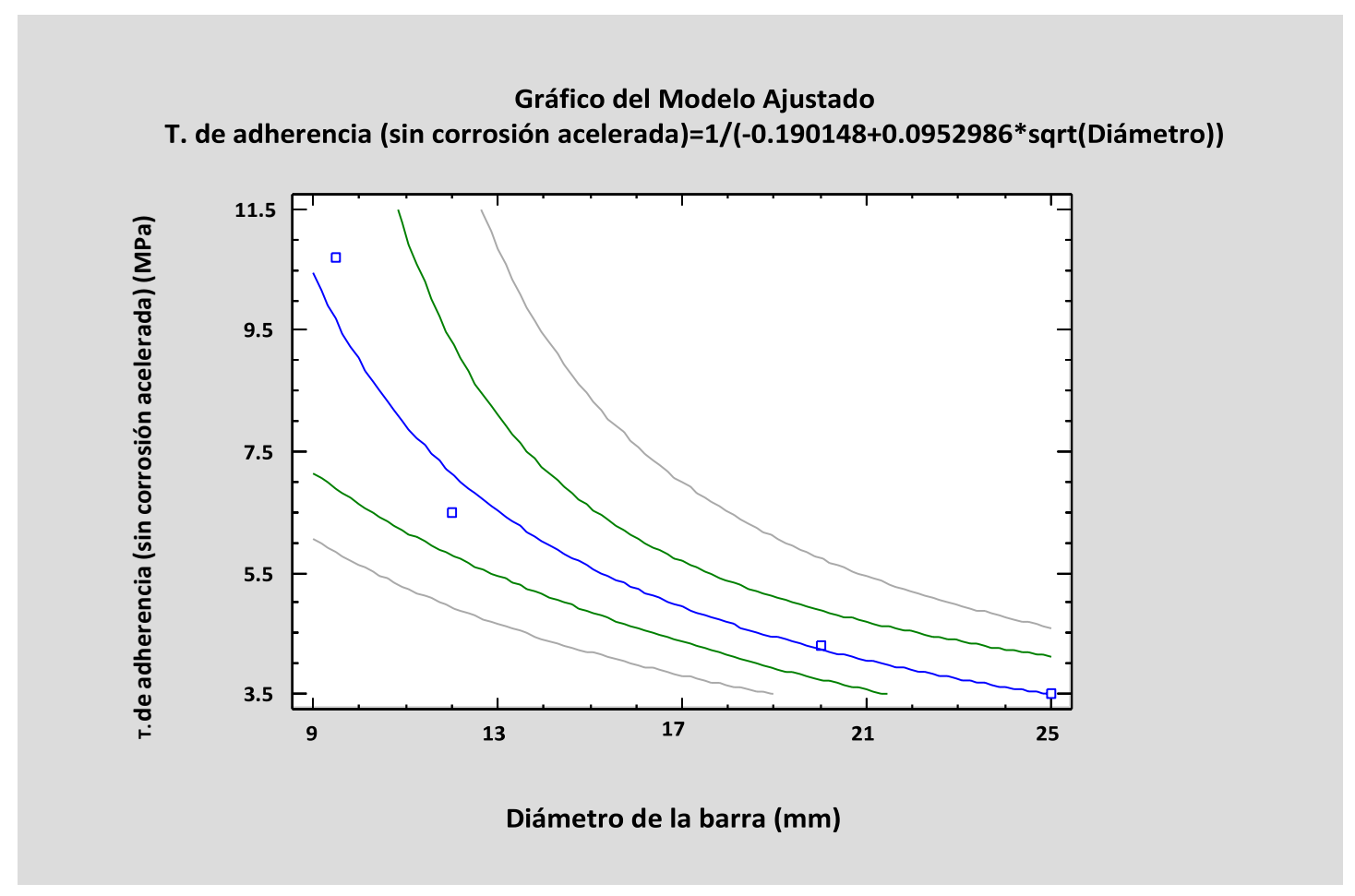

Figura 8. Tensión de adherencia (sin corrosión acelerada) vs. diámetro de barra (sin datos atípicos). Elaboración propia basada en resultados de STHATGRAPHICS 17.1 
Tensión máxima de adherencia diámetro

Empleando procedimientos de regresión simple (mínimos cuadrados) se construyó un modelo estadístico que describe el impacto del diámetro de la barra $(\mathrm{mm})$ sobre la tensión máxima de adherencia (MPa.). El modelo está representado en la Figura 9.

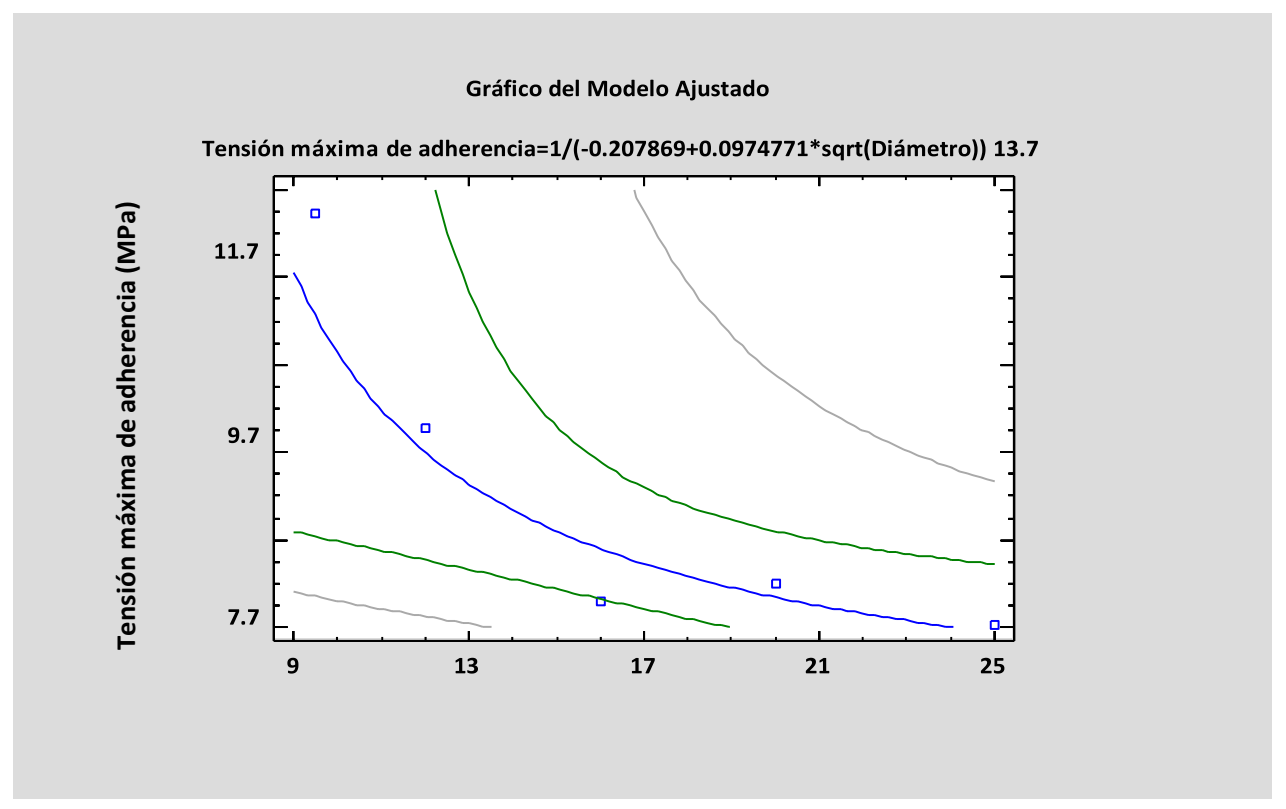

Figura 9. Tensión máxima de adherencia vs. Diámetro de barra Elaboración propia basada en resultados de STHATGRAPHICS 17.1

Una vez omitida la tensión máxima de adherencia identificada como atípica, se ajusta el modelo representado en la figura 10. Los parámetros estadísticos indican una mejor correlación entre la tensión máxima de adherencia y el diámetro de la barra con un nivel de confianza de $95.0 \%$
(Valor P=0.0008, coeficiente de correlación=0.999). Mediante el modelo ajustado se calcula el valor de tensión máxima de adherencia correspondiente a M-12; siendo igual a $5.92 \mathrm{MPa}$. Para un diámetro de $16 \mathrm{~mm}$. 


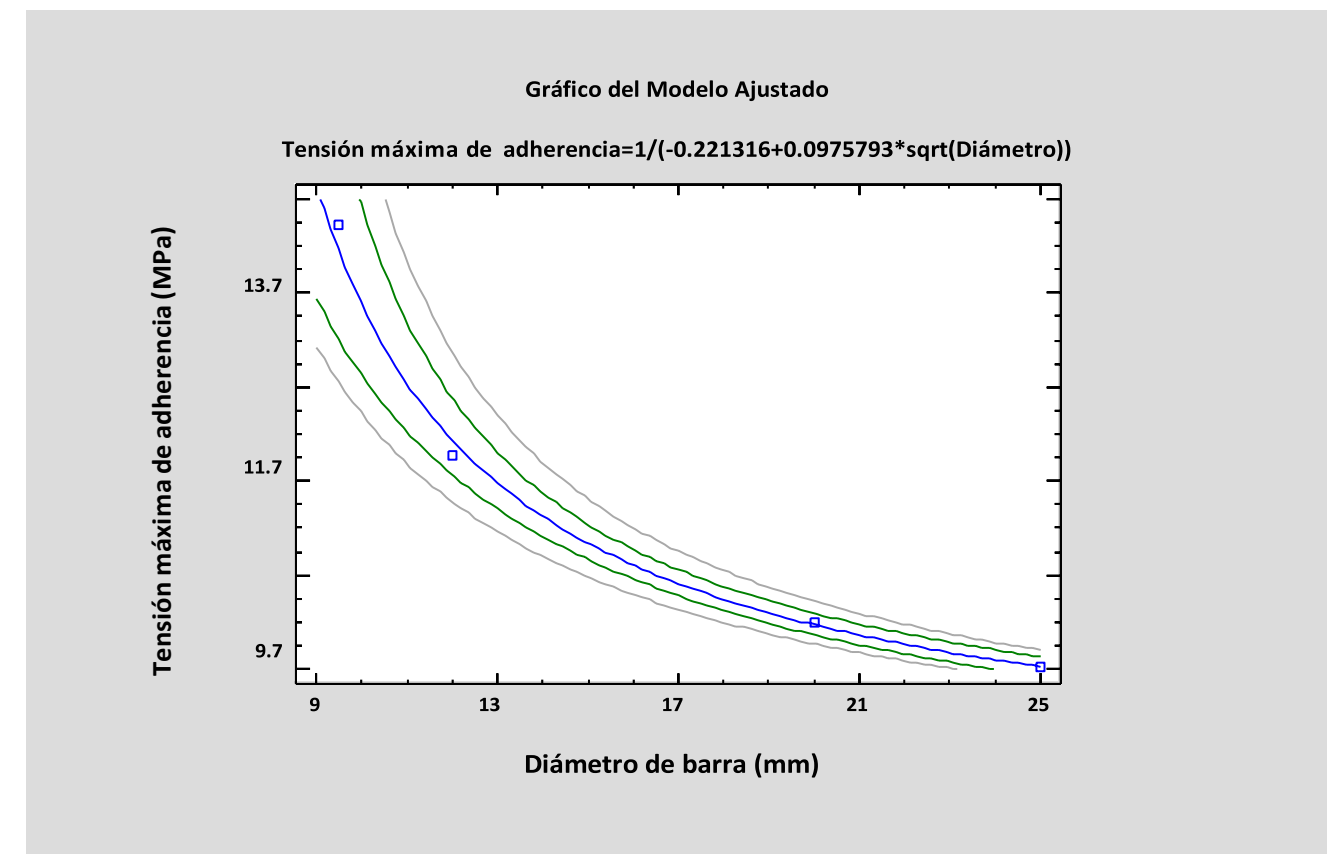

Figura 10. Tensión máxima de adherencia vs. diámetro de barra (sin datos atípicos). Elaboración propia basada en resultados de STHATGRAPHICS 17.1

\section{Comparación entre series}

Una vez realizado el análisis particular de cada serie se realiza la comparación para determinar la relación entre ellas. En la Figura 11 y tabla 9 se observa que existe una relación serial para la tensión de adherencia relacionada al diámetro y nivel de corrosión: la tensión de adherencia se reduce con el incremento de diámetro, con picos para niveles de corrosión bajos (100$120 \mathrm{~mm})$.

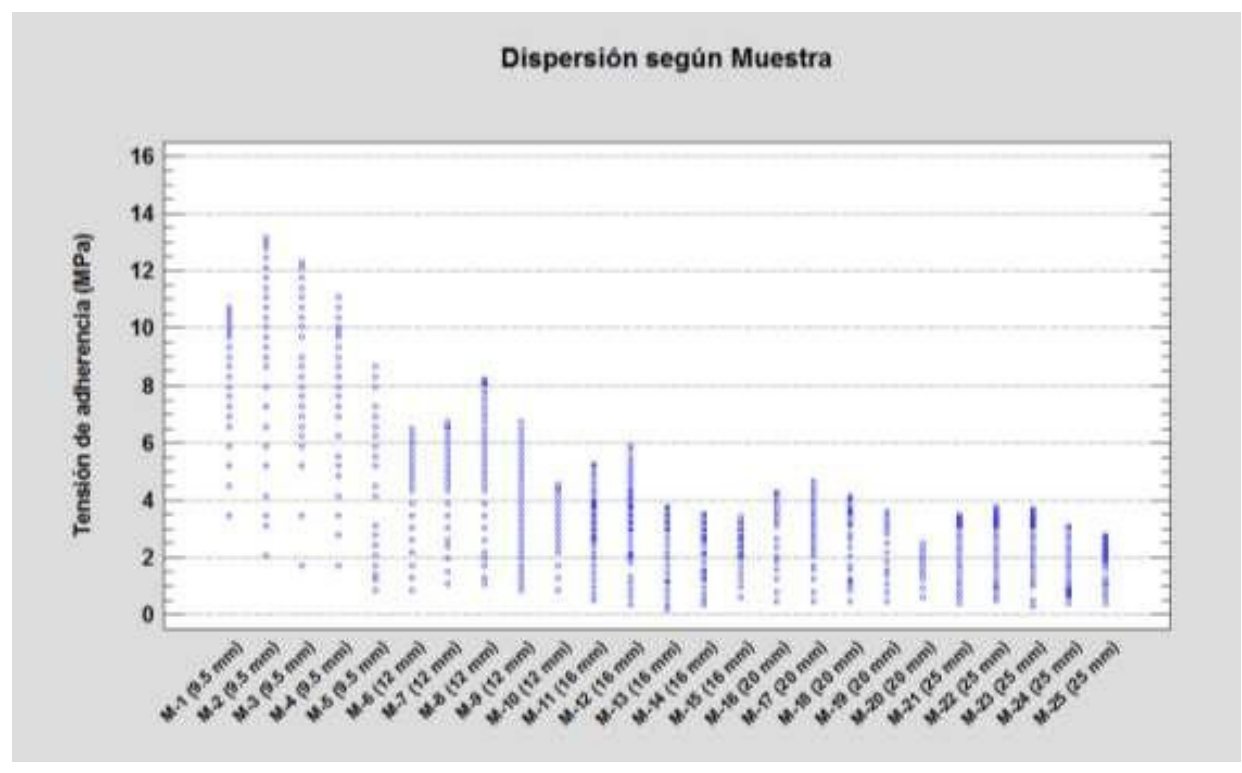

Figura 11. Dispersión de datos en la comparación se series experimentales Elaboración propia basada en resultados de STHATGRAPHICS 17.1 
Tabla 9. Análisis de varianza: comparación de series

\begin{tabular}{lccccc}
\hline Fuente & Suma de Cuadrados & Gl & Cuadrado Medio & Razón-F & Valor-P \\
\hline Entre grupos & 2404.25 & 24 & 100.177 & 32.61 & 0.0000 \\
Intra grupos & 1535.76 & 500 & 3.07151 & & \\
Total (Corr.) & 3940.0 & 524 & & & \\
\hline
\end{tabular}

Resultados de STHATGRAPHICS 17.1

En la tabla se descompone la varianza de los datos en dos componentes: un componente entre-grupos y un componente dentro-de-grupos. Puesto que el valor-P de la prueba-F es menor que 0.05 , existe una diferencia estadísticamente significativa entre las medias de las 25 variables con un nivel del 5\% de significación.

\section{Análisis de diseño de experimento}

El análisis de diseño de experimento permitió determinar el efecto del diámetro y del nivel de corrosión expresada como penetración promedio en la barra en la tensión de adherencia.

El diseño de experimento especificado constó de una variable respuesta (tensión de adherencia, medida en MPa.) y dos factores experimentales (diámetro de la barra medida en mm. y penetración promedio de corrosión expresada en mm.), el diseño tiene 25 ejecuciones.

El análisis de los efectos estimados para la tensión de adherencia permite concluir que la variable con mayor influencia (6.5083) es el diámetro de la barra, comparada con la penetración promedio de corrosión (-1.9101).

\section{Influencia de la pérdida parcial de} adherencia en la longitud de desarrollo

Cuando se proyecta un elemento de hormigón armado, uno de los aspectos que recibe especial atención es el diseño de la longitud de desarrollo, cuya finalidad de distribuir las tensiones producidas por las solicitaciones, en la barra de acero.

La adherencia está originada por dos tipos de causas, una de naturaleza físico química y otra de naturaleza mecánica donde interviene la longitud de desarrollo. La segunda en la cual se concentra este estudio, está constituido por la resistencia al deslizamiento debido a la penetración de la pasta de cemento en las irregularidades de la superficie de las barras de acero. Esta causa de origen mecánico que puede denominarse rozamiento, depende en gran parte de la longitud de desarrollo que tiene la barra dentro del concreto, así como el efecto de acuñamiento entre el hormigón y los corrugas de las barras.

Considerando lo expuesto, se comprueba que cuando se realizan adiciones de anticongelantes en el hormigón, se producen procesos corrosivos en las barras que degradan el comportamiento adherente; esto tiene como consecuencia la necesidad de incrementar la longitud de desarrollo.

\section{CONCLUSIONES}

Mediante el desarrollo de este estudio se logró determinar que:

El uso de aditivos anticongelantes en elementos de hormigón armado favorece la acción corrosiva en el acero; pues por su composición incorpora cloruros dentro de la mezcla de hormigón en porcentajes superiores al contenido máximo de iones de cloruro $(0,15 \%$ en peso de cemento para hormigón armado). La acción corrosiva se 
presenta de forma generalizada en las barras y dependerá de las condiciones de saturación del elemento.

Los diagramas de tensión de adherencia - deslizamiento en elementos de hormigón armado expresan los mecanismos de transferencia de tensiones en tres etapas: etapa de pseudo - adherencia, asociada a la adherencia química; etapa de deslizamiento de transición, caracterizada por el aplastamiento local y la microfisuración del hormigón; finalmente la etapa de fisuración interna, asociada al mecanismo de interacción mecánica.

El diámetro de la barra constituye el factor con efecto principal o de mayor impacto en la tensión de adherencia; se experimentan tensiones de adherencia mayores para una barra de menor diámetro pues en ella se desarrolla mayor superficie cobaricéntrica. Una sección de hormigón reforzada con varias barras de pequeño diámetro requiere una longitud de desarrollo menor que otra reforzada con barras de diámetro mayor con igual área de acero. Existe mayor eficiencia de barras pequeñas respecto a la falla por adherencia.

La naturaleza expansiva de los productos generados en el proceso corrosivo produce que la tensión de adherencia experimente incremento con el nivel de corrosión hasta un valor crítico de penetración promedio de $100 \mathrm{~mm}$., a partir del cual disminuye notablemente, debido a la construcción de una capa de productos de corrosión sueltos en la interface de la barra que deterioran el desempeño de la adherencia entre hormigón y acero.

\section{REFERENCIAS}

ACI224, C. (2007). ACI 224.1R-07 Causas, evaluación y reparación de Fisuras en estructuras de hormigón. American Concrete Institute

ACI306R-88, C. (2002). ACI 306R-88, Cold Weather Concreting.

ACI318S-14, A. C. I. (2014). Requisitos de Reglamento para Concreto Estructural. U.S.A

ArcelorMittal. (2016). Ficha técnica ARCELORMITTAL CA 50S. In L. Lomas (Ed.). Bolivia

Delgado, M. B. (2008). Modelización numérica del comportamiento adherente acero-hormigón en presencia de compresión transversal. (Tesis Doctoral), Universidad Politécnica de Valencia, Valencia

Fernández, M. (2003). Evaluación no lineal de los efectos estructurales producidos por las deformaciones diferidas del hormigón y el acero. (Tesis Doctoral), Escuela Técnica Superior de Ingenieros de Caminos, canales y puertos, Universidad Politécnica de Madrid, España

Fib, F. I. d. B. (2000). Bond of reinforcement in concrete: state-of-art report

García, M. (1998). Influencia de la corrosión en las propiedades mecánicas del acero. Hormigón y acero, 11-21

Hernández, J. J., y Hernández, J. A. (2010). Hormigón estructural. Diseño por estados límites (Parte I) (Vol. 1). Cuba

Li, C., y Zheng, J. (2005). Propagation of reinforcement corrosion in concrete and its effects on structural deterioration. Magazine of Concrete Research, 57(5), 261-271

Lundgren, K. (2007). Effect of corrosion on the bond between steel and concrete: an overview. Magazine of Concrete Research, 59, 447-461. doi: 10.1680/macr.2007.59.6.447

Molina, M., y Gutiérrez, J. (2008). Influencia de la degradación de la adherencia hormigón acero en la seguridad de las estructuras de hormigón armado. In U. C. D. B. o. Architecture (Ed.), (pp. 10). Madrid, España 
Prieto, M. (2014). Estudio de la adherencia de armaduras corroídas y su influencia en la capacidad resistente de elementos de hormigón armado. (Doctor Tesis Doctoral), Universidad Politécnica de Madrid, Madrid

RILEM/CEB/FIP, C. E. d. B. (1983). Recommendations on reinforcement steel for reinforced concrete. Revised Edition of RC-6: Bond test for reinforcement steel: Pull - out test. Paper presented at the Bulletin d'Information CEB, Bulletin d'Information 13

Ruiz, R., Lorefice, R., Etse, G., y Santillán, C. (2012). Análisis mesomecánico del fenómeno de adherencia en estructuras de hormigón armado. Mecánica Computacional, XXXI, 1749-1766

SENAMHI, S. N. d. M. e. H. (2013). Atlas Climatológico de Bolivia (pp. 1-243). La Paz, Bolivia
Simões, G. (2013). Estudio experimental de los efectos de la corrosión de las armaduras en vigas continuas de hormigón armado. (Tesis de Máster), Universitata Politecnica de Catalunya, Barcelona

Tepfers, R. (1973). A theory of bond applied to overlapped tensile reinforcement splices for deformed bars. (Tesis Doctoral), Chalmers University of Technology, Göteborg

Tórres, A. (2011). Structural consecuences of corrosion for reinforced concrete. Revista Técnica de Ingeniería Universidad Zulia., 34(2), 9

Zandi, K., Lundgren, K., y Coronelli, D. (2010). Severely corroded reinforced concrete with cover spalling: Part 2. Anchorage capacity. Joint Fib-RILEM Workshop on Modelling of Corrosion Concrete Structures 